\title{
Wind Turbine Power Generation: Response Prediction
}

\author{
Akash Deep Tyagi ${ }^{1}$, Ankush Yadav ${ }^{1}$, Nitin Joshi ${ }^{1}$ \\ ${ }^{\prime}$ (Suresh Gyan Vihar University, Mahal Jagatpura Jaipur Rajasthan)
}

\begin{abstract}
Abtract: The worldwide interest has been increasing about wind energy for power generation purpose due to continue increase in fuel cost and the need to have clean source of energy. Wind energy may enhance the power generation capabilities and maximize its capacity factor, inurn participate in generating power at lower cost. It has also been notice that renewable power generation through wind energy is also the fast growing energy technology. The optimization of the efficiency of wind turbine is prudent to complete the conventional power sources. Wind Turbine Power Generation (WTPG) is a complex phenomenon to understand since the real process has depends upon the Wind Velocity and the relative turbine dimensions and the outside climatological parameter like Wind Velocity (WV) nature of wind, etc. In this paper an effort has been made to develop a fuzzy logic approach to predict an appropriate WTPG considering the WV, AD and Chord Length of Turbine Blades (CLTB) as input parameters. The complexities of the parameters and the imprecision of linguistic expressions are taken into consideration; the application combining linguistic variable to optimize WTPG under multiple conditions is presented in this study.
\end{abstract}

Keywords: Wind Energy, Wind Power, Wind Velocity, Fuzzy Logic

\section{Introduction}

Wind energy generation has brought many challenges to the electrical power system engineers [1]. The problems encountered in the electrical network comprising wind energy systems are due to the continuous variations in the wind regime [2]. On-line estimation of wind turbine power coefficients using unknown input observer was reported by Odgaard P et al. [3]. In addition to conventional energy resources renewable energy can also play a vital role for energy demand of which wind energy is one of them. This is a kinetic energy associated with the atmospheric wind velocity. Wind energy for irrigation and milling has been used since the beginning of $20^{\text {th }}$ century. It is being used to generate electrical power also. The power carried by a flowing mass of air that is called wind is the product of the Cross-sectional area of the mass and the wind, the density of the wind, $\rho$, and the wind speed, $v$.

Where;

$$
\text { Wind Power, } P=\frac{1}{2} \times \rho \times A \times v^{3}
$$

$\begin{array}{lll}\rho & = & \text { Air density }\left(\mathrm{kg} / \mathrm{m}^{3}\right) \\ v & = & \text { wind velocity }(\mathrm{m} / \mathrm{s})\end{array}$

The air density is proportional to the air temperature and the air pressure; both vary with height above sea level. The power in the wind cannot be completely converted into mechanical energy of a wind turbine. Otherwise the air mass would be stopped completely in the intercepting rotor area and would cause a "congestion" of the cross-sectional area for the air masses. Theoretically maximum energy extraction from wind was discovered by Betz in 1926, and is written as

$$
\text { PBetz }=\frac{1}{2} \times \rho \times A \times v^{3} \times C p B e t z=\frac{1}{2} \times \rho \times A \times v^{3} \times 0.59
$$

Though this is not a hard and fast limit such as the Carnot efficiency it happens to be a useful rule-ofthumb estimate. According to Betz, even if no losses occurred, a wind turbine could utilize only $59 \%$ of the wind power. In addition when unavoidable swirl losses are included, this figure reduces to about 0.42 . This happens to be observed as the current limit of well-designed turbines today [4].

The competitive efficiency of the wind turbine is necessary to be an alternate the conventional power sources. Prediction of WTPG is a complex phenomenon to understand and is very difficult to present mathematically since the real process has the involvement of wind velocity and the relative turbine dimensions. Turbine Blades Hub above ground has the following phenomena, the higher the wind velocity (to the $1 / 7$ th power) and since power is proportional to the cube of the velocity, an increase in hub elevation from $30 \mathrm{~m}$ to $50 \mathrm{~m}$ leads to an Average wind speed 7.6\% higher. This becomes a significant cost-benefit trade-off since taller hubs become more expensive. Spacing of wind turbine on wind frames will prevent the maximum amount of wind to be intercepted. However, too close a spacing will lead to interference, and downwind units will be less productive [4]. Recent Progress about wind energy conversion has been reported by Musgrove, P.J. [5]. 


\section{Soft Computing Approaches}

Soft computing technology is an interdisciplinary research field in computational science. Presently various techniques of soft computing are being used for data analysis such as neural network, and fuzzy logic. For analytical purposes, it is considered that the fuzzy logic controller is a "black box" which receives inputs parameters and imprecision of linguistic expressions, whereas output is the prediction of desired resultant. Figure 1 shows the Black Box process of fuzzy controller.

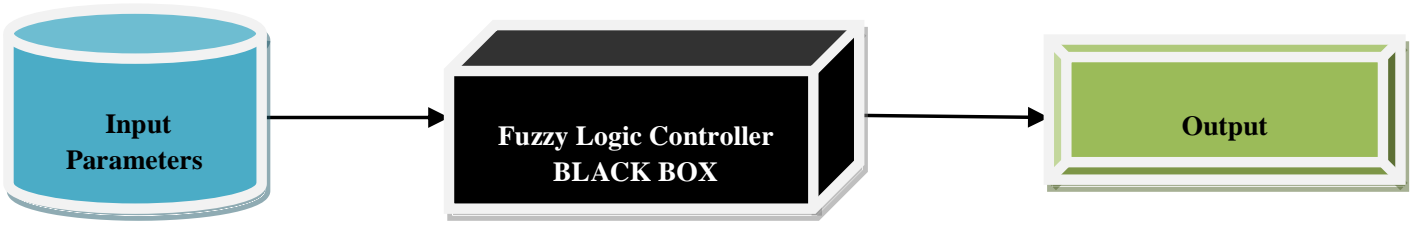

Figure 1: Black Box Process of Controller

In recent year the artificial intelligence have largely used in the different area including the transportation, agriculture, industrial application etc. In the power generation many expert system have been designed. Hossain et al have been reported an intelligent system using Fuzzy logic for the prediction of power generation [6]. A classical set theory can be extended to handle the partial membership enabling to express indistinct human concept using fuzzy set also describe by Kalogirou in 2003 [7]. A computational Model of a Novel, Two-Cup Horizontal Wind-Turbine System has been developed by Greg, M et al [8]. Recently a fuzzy logic controller for predicting wind turbine power generation has been reported by the Badrana et al [9] in which authors utilizes the fuzzy logic methodology to assess wind sites to decide which sites should be given the highest priority with respect to their benefits and costs, and to predict the annual generation for different turbines in the best sites. The criterion of evaluation using fuzzy logic is based on different parameters, i.e., wind resources, prevailing wind direction, above ground level (AGL), site capacity, soil conditions, site elevation, land cost, land roughness, temperature, cultural and environmental concerns, Aviation/telecommunications conflicts, nearby resident's concerns, site environmental issues (corrosion, humidity), and distance to transmission etc. Simulink model is developed to study the parameters that affect the power generation by wind turbines using MATLAB.

There are a number of different techniques that would work here and therefore a design choice must be made. Some of the techniques require a relatively accurate model of the system in order to develop a satisfactory system. Fuzzy Logic system, on the other hand, does not require a model of the system, instead, of that they depend the knowledge of an expert for the particular system. Therefore, with all of these kept in mind, a Fuzzy Logic expert system is introduced for the prediction of wind power generation from the wind turbine. The main advantage of Fuzzy Logic is that it can be tuned and adapted and if necessary, the degree of freedom of the system may be enhanced [10].

\section{Fuzzy Logic Modeling}

There are number of different methods are available some of the method require a comparatively truthful model in order to develop a satisfactory system. The concept of fuzzy set theory is a membership function, which represents numerically to what degree an element belongs to a set. In fuzzy set theory, an element can be a member of a particular set to a certain degree and at the same time be a member of a different set to a certain degree. To what degree an element belongs to a certain set is called the membership degree. In fuzzy rule-based systems, knowledge is represented by if-then rules. Fuzzy rules consist of two parts: an antecedent part stating conditions on the input variable(s) and a consequent part describing the corresponding values of the output variable(s) [11]. The aim of this study was to construct fuzzy knowledge-based models for the prediction of the power generation by controlling wind velocity of wind turbine (based on the Mamdani) approach. Fuzzy modeling involves the following three steps

$\begin{aligned} \text { i. } & \text { Fuzzification } \\ \text { ii. } & \text { Fuzzy Rule Inference } \\ \text { iii. } & \text { Defuzzification }\end{aligned}$

\subsection{Fuzzification}

The fuzzification comprises the process of transforming crisp values into grades of membership for linguistic terms of fuzzy sets. The Membership Function (MF) is used to associate a grade to each linguistic term. The terminology of linguistic variable was introduced by Zadeh [12] as an approach to capture natural experience commonly used by controller black box. 


\subsection{Fuzzy Rule Inference}

Fuzzy rule inference method is used to define some sets of fuzzy logic operators along with production rules. The most common rule is called IF-THEN Rule, which can be used to formulate the conditional statements that comprise fuzzy logic.

\subsection{Defuzzification}

In Defuzzification, Fuzzy sets act as the input in the process and output is the single number. The aggregate of fuzzy set encompasses a range of output values and so must be defuzzified in order to get the single output value from the set. A Conceptual Fuzzy Logic Modeling Approach has been depicted in Figure 2.

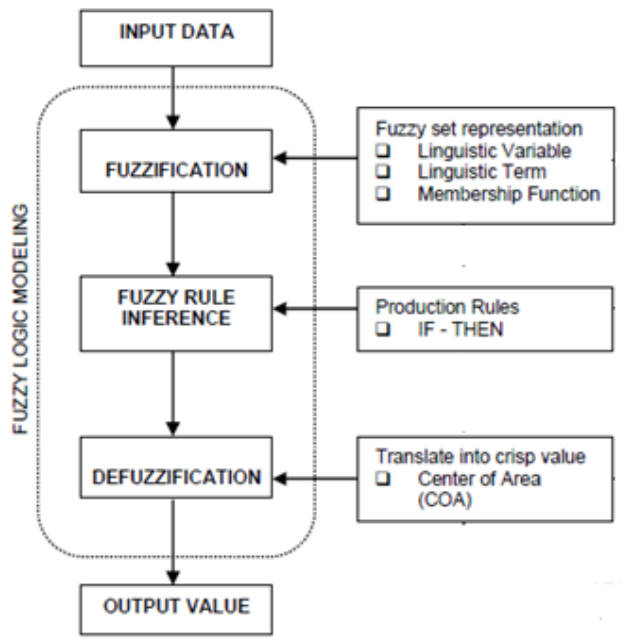

Figure 2: Conceptual Fuzzy Logic Modeling

\section{FUZZY INFERENCE ENGINE (FIE)}

Fuzzy inference is the process of formulating the mapping from a given input set to an output using fuzzy logic. Fuzzy logic [11]-[13] is a superset of conventional Boolean logic and extends it to deal with new aspects such as partial truth and uncertainty. The basic elements of fuzzy logic are linguistic variables, fuzzy sets, and fuzzy rules [14]. The linguistic variables' values are words, specifically adjectives like "very low", "low", "medium", "high", and "very high" A fuzzy set is a collection of couples of elements. It generalizes the concept of a classical set, allowing its elements to have a partial membership. The degree to which the generic element " $\mathrm{x}$ " belongs to the fuzzy set A (expressed by the linguistic statement $\mathrm{x}$ is A) is characterized by a membership function (MF), fA(x). The membership function of a fuzzy set corresponds to the indicator function of the classical sets. It can be expressed in the form of a curve that defines how each point in the input space is mapped to a membership value or a degree of truth between 0 and 1 . The most common shape of a membership function is triangular, although trapezoidal and bell curves are also used. This operation normalizes all inputs to the same range and has a direct effect on system performance and accuracy.

A fuzzy set " $A$ " defined within a finite interval called universe of discourse $U$ as follows:

$\mathrm{A}=\{(\mathrm{x}, \mathrm{fA}(\mathrm{x})), \mathrm{fA}(\mathrm{x}): \mathrm{U} \rightarrow[0,1]\}$

$\mathrm{U}$ is the whole input range allowed for a given fuzzy linguistic variable. All fuzzy sets related to a given variable make up the term set, the set of labels within the linguistic variable described or, more properly, granulated. Fuzzy rules form the basis of fuzzy reasoning. They describe relationships among imprecise, qualitative, linguistic expressions of the system's input and output. Generally, these rules are natural language representations of human or expert knowledge and provide an easily understood knowledge representation scheme.

A typical conditional fuzzy rule assumes a form such as

IF Load is "High" AND Backup is "Poor" THEN Performance is "Not satisfactory".

Load is "High" and Backup is "Poor" is the rule's premise; while Performance is "Not Satisfactory" is the consequent. The premise predicate might not be completely true or false, and its degree of truth ranges from 0 to 1. We compute this value by applying the membership functions of the fuzzy sets labeled "High" and "Poor" to the actual value of the input variables Load and Backup. After that, fuzzification is applied to the conclusion; the way in which this happens depends on the inference model.

There are two types of fuzzy inference models:

1. Mamdani [15], 
2. TSK or Sugeno [16].

Interpreting an if-then rule involves two distinct parts: first evaluating the antecedent and then applying results to the consequent (known as implication) [17]-[18]. In the case of two-valued or binary logic, if-then rules do not present much difficulty. If the premise is true, then the conclusion is true, whereas with fuzzy approach, if the antecedent is true to some degree of membership, then the consequent is also true to that same degree.

Mamdani-type [16] inference expects the output membership functions to be fuzzy sets. After the aggregation process, there is a fuzzy set for each output variable that needs defuzzification. It is possible, and in many cases much more efficient, to use a single spike as the output's membership function rather than a distributed fuzzy set. This is sometimes known as a singleton output membership function, and it can be thought of as a pre-defuzzified fuzzy set. It enhances the efficiency of the defuzzification process because it greatly simplifies the computation required by the more general Mamdani method, which finds the centroid of a twodimensional function. Rather than integrating across the two-dimensional function to find the centroid, Sugenotype systems use weighted sum of a few data points. In general, Sugeno-type systems can be used to model any inference system in which the output membership functions are either linear or constant.

\section{Proposed Model}

As shown in Fig. 3, the three input parameter WV, AD and CLTB are considered for the prediction of output WTPG; the fuzzy logic toolbox of MATLAB was used. For fuzzification of these factors the linguistic variables Very Low (VL), Low (L), Medium (M), High (H) and Very High $(\mathrm{VH})$ were used for the input and output. For the study, the bisector method for defuzzification was used because these operators assure that the vertical line divides the region into two sub-regions of equal area of the output between the rules. The units of the used factors were WV $(\mathrm{m} / \mathrm{s})$, AD $(\mathrm{kg} / \mathrm{cubic}$ meter) and CLTB $(\mathrm{m})$, and WTPG $(\mathrm{W})$. With the fuzzy sets defined, it is possible to associate the fuzzy sets in the form of fuzzy rules. For the three inputs and one output, a fuzzy rule is developed. The main advantage of Fuzzy Logic is that it can be tuned and adapted if necessary, thus enhancing the degree of freedom of the system Rajagopalan et al., 2003 [10].

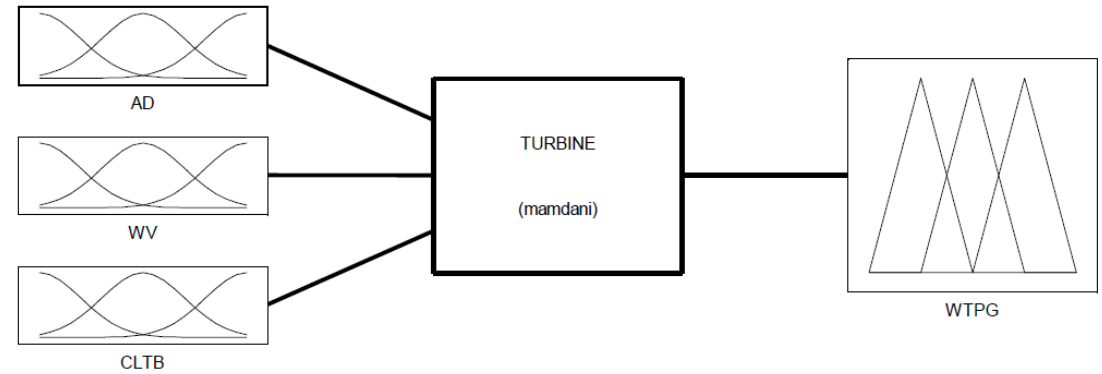

Figure 3: Inference system Block Diagram

\subsection{Membership Functions}

For the $\mathrm{f}_{\mathrm{A}}(\mathrm{x})$ as the Membership Function (MF), a large class of functions can be taken viz. triangular, trapezoidal, Gaussian and bell functions. However we have selected Gaussian function. MFs considered for AD, WV, CLTB and WTPG are represented by 5 Gaussian curves for this model illustrated in Fig. 4, 5, 6 and 7 .

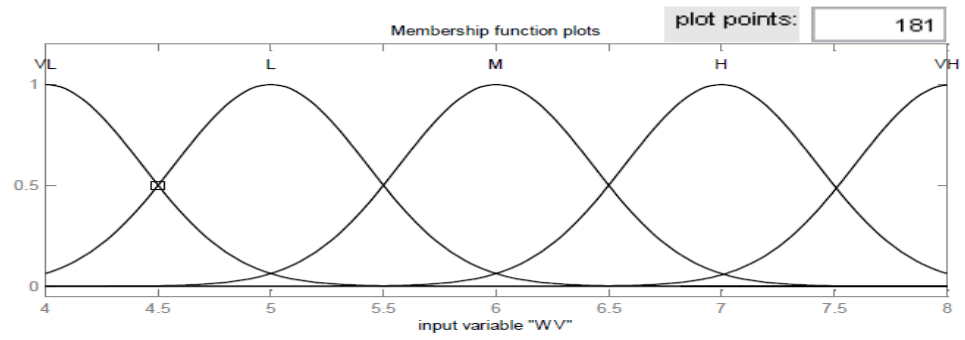

Figure 4. Membership Functions for the corresponding to WV 


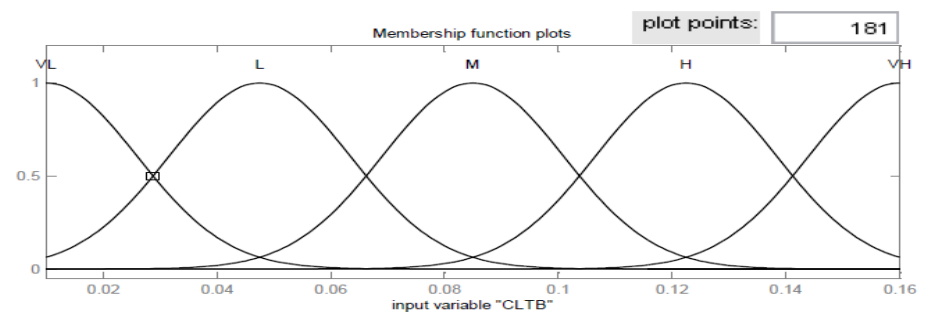

Figure 5. Membership Functions for the corresponding to CLTB

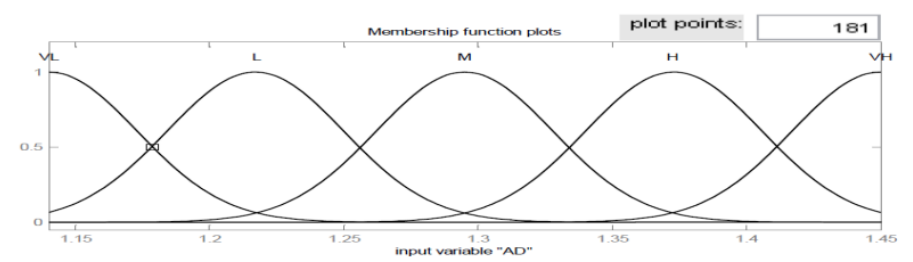

Figure 6. Membership Functions for the corresponding to AD

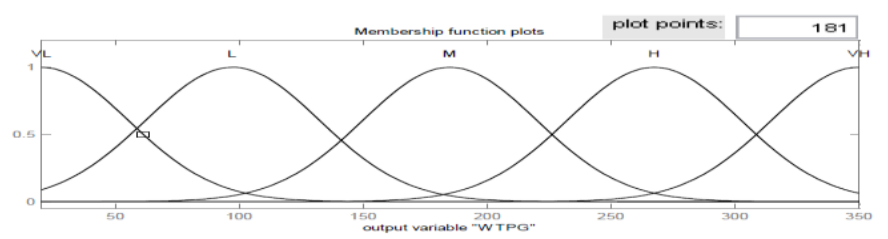

Figure 7. Membership Functions for the corresponding to WTPG

When multiple input combinations are to get executed, the basic concept of switching and time slices comes into picture. In our proposed algorithm as shown under, a newly WV (), AD () and CLTB() value, will be added to the input queue. This queue consists of the remaining input values from last cycle that has not yet been executed

Loop

For each state of the WTPG, do the following:

1. For each value of power generation, feed its $A D, W V$ and CLTB into the inference engine. Consider the output of inference module as WTPG.

2. Store the values of WTPG in an array WTPG ().

3. Execute the corresponding WTPG () value of highest magnitude until a scheduling event occurs.

4. Update the system states.

End Loop

\section{Results and Discussion}

Fuzzification of the used parameter is made by aid follows functions. These formulas were determined by using assumed values.

$A D\left(i_{1}\right)=\left\{\begin{array}{c}i_{1} ; 1.14 \leq i_{1} \leq 1.45 \\ 0 ; \text { otherwise }\end{array}\right\}$

$W V\left(i_{2}\right)=\left\{\begin{array}{c}i_{2} ; 4 \leq i_{2} \leq 8 \\ 0 ; \text { otherwise }\end{array}\right\}$

$\operatorname{CLTB}\left(i_{3}\right)=\left\{\begin{array}{c}i_{3} ; 0.01 \leq i_{3} \leq 0.16 \\ 0 ; \text { otherwise }\end{array}\right\}$

$\operatorname{WTPG}\left(o_{1}\right)=\left\{\begin{array}{c}o_{1} ; 20 \leq o_{1} \leq 350 \\ 0 ; \text { otherwise }\end{array}\right\}$

$\mathrm{AD}, \mathrm{WV}, \mathrm{CLTB}$ and WTPG are set up as shown in 4 to 7 . The membership values used for the FIE were obtained from the above formulas. These membership functions helped in converting numeric variables into linguistic terms. For example, the linguistic expressions and membership functions for wind velocity $(W V)$ obtained from the developed rules and above the formula are given as following.

The Gaussian fuzzy set is defined as:

$\operatorname{gaussian}(x ; c, \sigma)=e^{-\frac{1}{2}\left(\frac{x-c}{\sigma}\right)^{2}}$ 
Where $\mathrm{c}$ represents the MFs centre and $\sigma$ determines the MFs width.

$$
\begin{aligned}
& \mu_{V L}(x)=e^{-\frac{1}{2}\left(\frac{x-c}{\sigma}\right)^{2}} \text {, where } \mathrm{c}=4, \sigma=0.427
\end{aligned}
$$

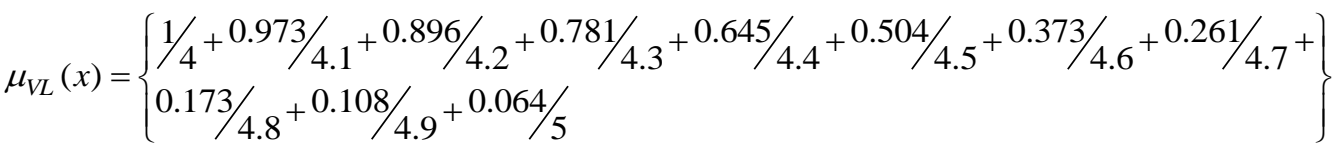

$$
\begin{aligned}
& \mu_{L}(x)=e^{-\frac{1}{2}\left(\frac{x-c}{\sigma}\right)^{2}}, \text { where } \mathrm{c}=5, \sigma=0.427
\end{aligned}
$$

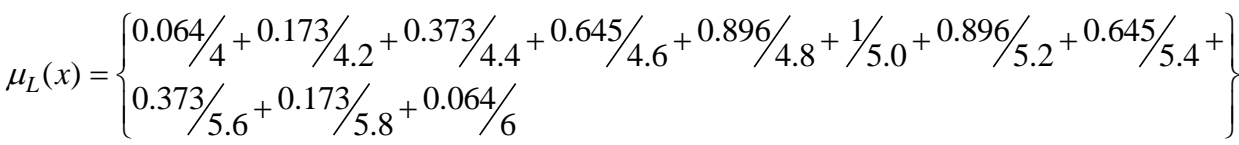

$$
\begin{aligned}
& \mu_{M}(x)=e^{-\frac{1}{2}\left(\frac{x-c}{\sigma}\right)^{2}}, \text { where } \mathrm{c}=6, \sigma=0.427
\end{aligned}
$$

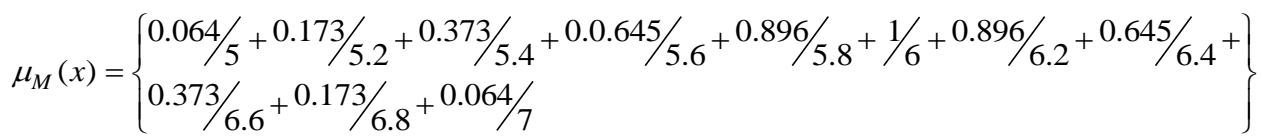

$$
\begin{aligned}
& \mu_{H}(x)=e^{-\frac{1}{2}\left(\frac{x-c}{\sigma}\right)^{2}}, \text { where } \mathrm{c}=7, \sigma=0.427 \\
& \mu_{H}(x)=\left\{\begin{array}{l}
0.064 / 6+0.173 / 6.2+0.373 / 6.4+0.645 / 6.6^{+0.896 / 6.8}+1 / 7+0.896 / 7.2+0.645 / 7.4+ \\
0.373 / 7.6+0.173 / 7.8+0.064 / 8
\end{array}\right\} \\
& \mu_{V H}(x)=e^{-\frac{1}{2}\left(\frac{x-c}{\sigma}\right)^{2}}, \text { where } \mathrm{c}=8, \sigma=0.427 \\
& \mu_{V H}(x)=\left\{0.064 / 7+0.173 / 7.2^{+0.373 / 7.4}+0.645 / 7.6^{+0.896 / 7.8}+1 / 8\right\}
\end{aligned}
$$

The determination of conclusion is taken when the rules that are applied to deciding what the wind power generation to the plant (wind turbine) should be. For this the recommendations from all the rules are combined to determine the wind power generation input to the wind turbine.

The composition of two fuzzy relations is defined as follows:

Let $\mathrm{P}$ be a fuzzy relation from $\mathrm{X}$ to $\mathrm{Y}$ and $\mathrm{Q}$ be a fuzzy relation from $\mathrm{Y}$ to $\mathrm{Z}$ such that the membership degree is defined by $\mathrm{P}(\mathrm{x}, \mathrm{y})$ and $\mathrm{Q}(\mathrm{y}, \mathrm{z})$. Then, a third fuzzy relation $\mathrm{R}$ from $\mathrm{X}$ to $\mathrm{Z}$ can be produced by the composition of $\mathrm{P}$ and $\mathrm{Q}$, which is denoted as PoQ. Fuzzy relation R is computed by the formula

$$
\begin{aligned}
& \mathrm{R}(\mathrm{x}, \mathrm{z})=(\mathrm{P} \circ \mathrm{Q}) \\
& (\mathrm{x}, \mathrm{z})=\max \{\min [\mathrm{P}(\mathrm{x}, \mathrm{y}), \mathrm{Q}(\mathrm{y}, \mathrm{z})]\}
\end{aligned}
$$

For example, for $\mathrm{AD}=1.295 \mathrm{~kg} /$ cubic meter, $W V=6.5 \mathrm{~m} / \mathrm{s}$ and CLTB $=0.1 \mathrm{~m}$, the rules $28,29,33$ and 34 will be applied which notices that $\mu_{M}(A D)=1$ but that the other membership functions for the $A D$ inputs are all "off" (i.e., their values are zero). For the $W V$ input it is seen that $\mu_{M}(W V)=0.5$ and $\mu_{H}(W V)=0.5$ and that the other membership functions are off. Again for the CLTB input it is seen that $\mu_{M}(C L T B)=0.5$ and $\mu_{H}(C L T B)=$ 0.5 and that the other membership functions are off.

\subsection{For rule 28}

The premise $(M)$, we have $\mu_{M}(28)=\min \left\{\mu_{M}(A D), \mu_{M}(W V), \mu_{M}(C L T B)\right\}=\min \{1,0.5,0.5\}=0.5$, by using the minimum of the three membership values. The notation $\mu_{28}$ represents for rule (28) so that we are 0.5 certain that this rule applies to the current situation. This rule indicates that if its premise is true then the action indicated by its consequent should be taken. For rule (28) the main consequent is "wind power is medium". The membership function for the conclusion reached by rule (28), which is denoted as $\mu_{28}$, is given by $\mu_{28}(W T P G)=$ $\min \left\{0.5, \mu_{M}(W T P G)\right\}$. This membership function defines the implied fuzzy set for rule (28).

\subsection{For Rule 29}

The premise $(M)$, we have $\mu_{M}(29)=\min \left\{\mu_{M}(A D), \mu_{M}(W V), \mu_{H}(C L T B)\right\}=\min \{1,0.5,0.5\}=0.5$, by using the minimum of the three membership values. The notation $\mu_{29}$ represents for rule (29) so that we are 0.5 certain that this rule applies to the current situation. This rule indicates that if its premise is true then the action 
indicated by its consequent should be taken. For rule (29) the main consequent is "wind power is medium". The membership function for the conclusion reached by rule (29), which is denoted as $\mu_{29}$, is given by $\mu_{29}(W T P G)=$ $\min \left\{0.5, \mu_{M}(W T P G)\right\}$. This membership function defines the implied fuzzy set for rule (29).

\subsection{For rule 33}

The premise $(H)$, we have $\mu_{H}(33)=\min \left\{\mu_{M}(A D), \mu_{H}(W V), \mu_{M}(C L T B)\right\}=\min \{1,0.5,0.5\}=0.5$, by using the minimum of the three membership values. The notation $\mu_{33}$ represents for rule (33) so that we are 0.5 certain that this rule applies to the current situation. This rule indicates that if its premise is true then the action indicated by its consequent should be taken. For rule (33) the main consequent is "wind power is High". The membership function for the conclusion reached by rule (33), which is denoted as $\mu_{33}$, is given by $\mu_{33}(W T P G)=$ $\min \left\{0.5, \mu_{H}(W T P G)\right\}$. This membership function defines the implied fuzzy set for rule (33).

\subsection{For rule 34}

The premise $(H)$, we have $\mu_{H}(34)=\min \left\{\mu_{M}(A D), \mu_{H}(W V), \mu_{H}(C L T B)\right\}=\min \{1,0.5,0.5\}=0.5$, by using the minimum of the three membership values. The notation $\mu_{34}$ represents for rule (34) so that we are 0.5 certain that this rule applies to the current situation. This rule indicates that if its premise is true then the action indicated by its consequent should be taken. For rule (34) the main consequent is "wind power is High". The membership function for the conclusion reached by rule (34), which is denoted as $\mu_{34}$, is given by $\mu_{34}(W T P G)=$ $\min \left\{0.5, \mu_{H}(W T P G)\right\}$. This membership function defines the implied fuzzy set for rule (34).

It is noticed that we are certain that all rules (28), (29), (33) and (34) apply to the current situation. From Mamdani max-min inference, the membership function of system will be found as max $\left(\mu_{28}, \mu_{29}, \mu_{33}, \mu_{34}\right)$ $=0.5$ and then defuzzification operation is applied to the final component of the fuzzy controller produced by the inference mechanism and combines their effects to provide the "most certain" controller output (plant input). Then the output denoted by "WTPG ${ }^{\text {crisp " }}$ " can be calculated that best represents the conclusions of the fuzzy controller that are represented with the implied fuzzy sets.

The Bisection for defuzzification is being used to know the output membership values whereas, the value of membership functions are multiplied by their corresponding singleton values and then the result is divided by the sum of the membership value. The bisection of "WTPG $G^{\text {crisp" }}$ can be computed by the flowing formula:

$$
W T P G^{\text {crisp }}=\frac{\sum_{i} a_{i} \mu_{(i)}}{\sum_{i} \mu_{(i)}}
$$

Where, $a_{i}$ is the position of the singleton in i the universe, and $\mu_{(i)}$ is the corresponding membership function.

For the rules 28, 29, 33 and 34 we have $a_{28}=175, a_{29}=175, a_{33}=262.5, a_{34}=262.5$

$$
W T P G^{\text {crisp }}=\frac{175 * 0.5+175 * 0.5+262.5 * 0.5+262.5 * 0.5}{0.5+0.5+0.5+0.5}=218.75
$$

The diagram of the whole computational fuzzy modeling is presented in Figure 8.
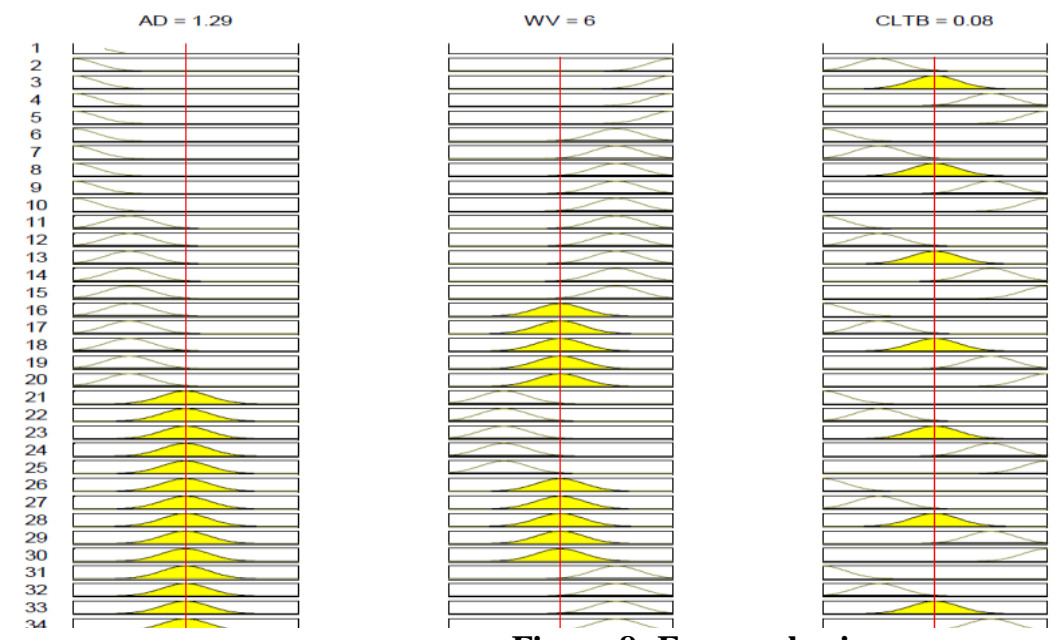

$W T P G=175$

Figure 8: Fuzzy rule viewer

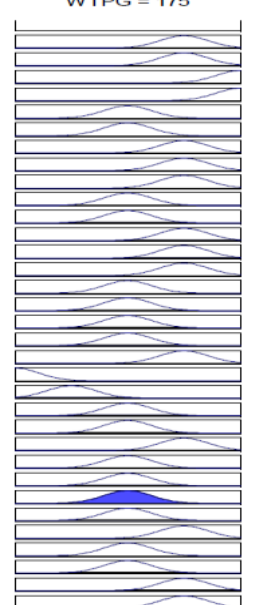


Once all the variables of WV, and CLTB get executed by the FIE, a decision surface can generate showing the value of the output variable WTPG as shown in figure 9. The decisions surface is the dynamic combination of WV, and CLTB affecting WTPG. Figure 9 present a three-dimensional curve representing the mapping from WV and CLTB as inputs (axis X and Y) and WTPG as an output (axis Z). In varying conditions of WV, and CLTB parameters, the maximum value of WTPG likely to be changes accordingly that depends on the inference engine mechanism. That is wind power generation increased as increase in wind velocity depicted in figure 10.

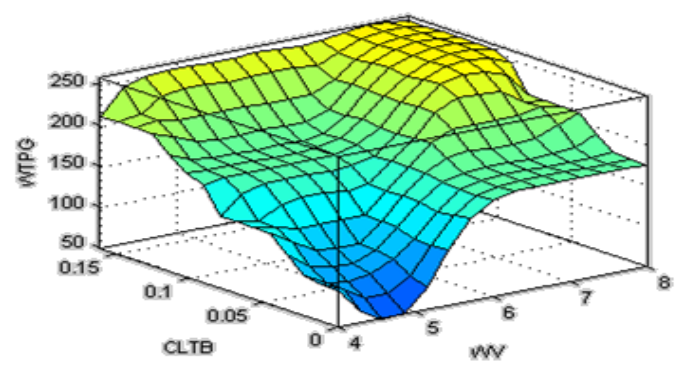

Figure 9: Decision Surface

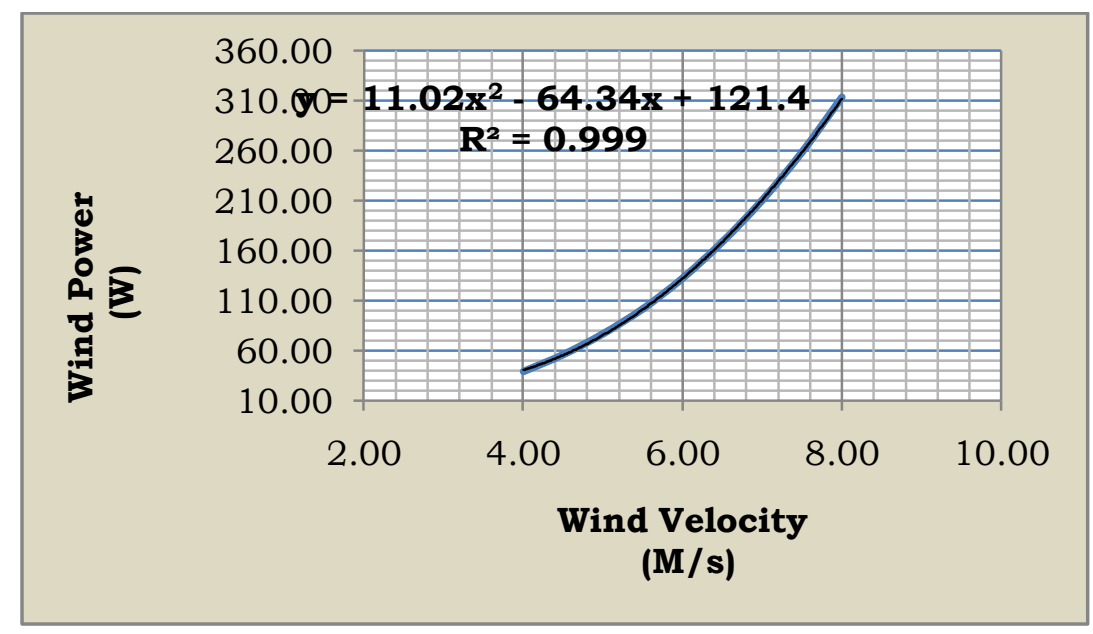

Figure 10: Wind power generation v/s wind velocity

\section{Conclusion}

In this paper an effort has been made to develop a fuzzy logic approach for the prediction of appropriate WTPG considering the WV, AD and Chord Length of Turbine Blades (CLTB) as input parameters which is necessary for the current renewable energy utilization in many countries particularly in the rural areas. In comparison to other predictive modeling techniques, fuzzy models have the advantage of being simple due to the Fuzzy Inference System(FIS) capability to deal with problems that are based on user knowledge and experience. This system can be developed further by increasing the knowledge rules and by addition of GeneticFuzzy and Neuro-Fuzzy to the system

\section{Acknowledgement}

It is our pleasure and privilege to acknowledge and express our deep sense of gratitude to our teacher and guide, Mr. Amit Mandal and co-guide Dr. Pradeep Yadav. They inspired and guided us every step towards achieving this goal, despite their busy academic schedule.

\section{References}

[1]. Rahman Ataur, Rahman Mozasser, Hasan S.K. and Hossen Jakir, (2009)," Prediction of Power Generation of Small Scale Vertical Axis Wind Turbine Using Fuzzy Logic", Journal of Urban and Environmental Engineering, 3(2), 43-51

[2]. Laks J., Pao L. and Wright, (2009) "Control of Wind Turbines: Past, Present and Future", ACC June 2009.

[3]. Odgaard P., Damgaard C. and Nielsen, (2008) "On-Line Estimation of Wind Turbine Power Coefficients Using Unknown Input Observers”, IFAC, Seoul, Korea, Jul 6-11, 2008.

[4]. Mallet Victor K., (2001) "The Use of Wind Energy in India - Lessons learned", Term Paper, Sustainable Energy, 10.391J, Spring 2001

[5]. Musgrove, P.J. (1987) Wind Energy Conversion: Recent Progress and Future Prospects. Solar and Wind Technology, 4(1), 37-49 
[6]. Hossain Altab, Rahman Ataur, Rahman Mozasser, Hasan S.K. and Hossen Jakir, (2009), Prediction of Power Generation of Small Scale Vertical Axis Wind Turbine Using Fuzzy Logic", Journal of Urban and Environmental Engineering, 3(2), 43-51

[7]. Kalogirou, S.A. (2003) Artificial intelligence for the modeling and control of combustion processes: a review. Prog Energy Combust Sci. 29(5), 515-566.

[8]. Greg, M., Robert, E., \& Abraham, J. (2009) "Computational Model of a Novel, Two-Cup Horizontal Wind-Turbine System" The Open Mechanical Engineering Journal, J. 3, 26-34.

[9]. Badrana O., Abdulhadib E. and El-Tousc Y,(2011) "Fuzzy Logic Controller for Predicting Wind Turbine Power Generation", International Journal of Mechanical and Materials Engineering (IJMME), 6 (2011), No.1, 51-66

[10]. Rajagopalan, A., Washington, G., Rizzani, G. \& Guezennec, Y. (2003) Development of fuzzy logic and neural network control and advanced emissions modeling for parallel hybrid vehicles, Center for Automotive Research, Intelligent Structures and Systems Laboratory, Ohio State University, U.S.A.

[11]. Zadeh L. A., "Fuzzy logic, neural networks, and soft computing," Commun. ACM, vol. 37, pp. 77-84, March 1994.

[12]. Ross, J.T. (1995) Fuzzy Logic with Engineering Applications, New York: McGraw hill Inc., U.S.A.

[13]. L. A. Zadeh, "Fuzzy sets versus probability," Proc. IEEE, vol. 68, pp. 421-421, March 1980.

[14]. W. Pedrycz and F. Gomide, An introduction to fuzzy sets: analysis and design: The MIT Press, 1998.

[15]. E. H. Mamdani, "Application of fuzzy algorithms for the control of a dynamic plant," Proc. IEE, vol. 121, pp. 1585-1588, Dec 1974 .

[16]. T. Takagi and M.Sugeno, "Fuzzy identification of systems and its applications to modeling and control," IEEE Trans. Syst., Man, Cybern., vol. 15, pp. 116-132, 1985.

[17]. H. Surmann and A. P. Ungering, "Fuzzy rule-based systems on general-purpose processors," IEEE Micro, vol. 15, pp. 40-48, Aug 1995.

[18]. G. Ascia and V. Catania, "A general purpose processor oriented to fuzzy reasoning," in Proc. 10th IEEE International Conf. Fuzzy Systems, Melbourne, Australia, 2001, pp. 352-35 